\title{
A COMPOSIÇÃO DOS BANDOS MISTOS DE AVES NA MATA ATLÂNTICA DA SERRA DE PARANAPIACABA, NO SUDESTE BRASILEIRO
}

\author{
MACHADO, C. G. \\ Departamento de Ciências Biológicas, Universidade Estadual de Feira de Santana, BR 116, \\ Km 03, CEP 44031-460, Feira de Santana, BA \\ Correspondência para: Caio Graco Machado, Departamento de Ciências Biológicas, Universidade Estadual de Feira \\ de Santana, BR 116, Km 03, CEP 44031-460, Feira de Santana, BA, e-mail: cgraco@zaz.com.br \\ Recebido em 22/01/98 - Aceito em 14/08/98 - Distribuído em 23/02/99
}

(Com 3 figuras)

\section{ABSTRACT \\ Mixed flocks of birds in Atlantic Rain Forest in Serra de Paranapiacaba, southeastern Brazil}

Mixed flocks of birds are two or more species groupings whose formation and cohesion depend on behavioral responses among members in the group. This study investigated the composition of mixed flocks in Atlantic Rain Forest at Parque Estadual Intervales (2412' a $24^{\circ} 25^{\prime}$ 'S; 48 $03^{\prime}$ a $48^{\circ} 30^{\prime} \mathrm{W}$ ), in Serra de Paranapiacaba high elevation, SP, Brazil. Field work was conduced from April 1990 to March 1991. Flock size, species composition and high distribuition in vegetation were recorded for each mixed flock. Number of bird species per flocks was positively correlated with flock size ( $r=$ 0,8). A total of 120 bird species were registered in 388 records of flocks; only three species were categorized regular species: Basileuterus culicivorus, Philydor rufus e Sittasomus griseicapillus. The species and flock size averaged numbers, respectively 6,71 and 17,26 birds per flock. The numbers, however, showed significant seasonal variation. During the wet season (November to March) mixed flocks presented their richest number of species and greatest number of individuals.

Key words: mixed flocks of birds, Atlantic Rain Forest, foraging behaviour.

\section{RESUMO}

Bandos mistos de aves são agrupamentos de duas ou mais espécies cuja formação e coesão se devem a interações comportamentais entre seus integrantes. Este estudo investigou a composição dos bandos mistos na Mata Atlântica do Parque Estadual Intervales, no alto da Serra de Paranapiacaba, SP, Brasil (241' a 2425' S; 4803' a 48³0'W). Foram feitas visitas mensais de abril de 1990 a março de 1991. A cada bando contatado registrava-se o número de indivíduos, composição e distribuição específica dentro dos bandos e na vegetação. Em 388 contatos com bandos mistos, registrou-se um total de 120 espécies de aves, com apenas três espécies regulares: Basileuterus culicivorus, Philydor rufus e Sittasomus griseicapillus. O número de indivíduos e de espécies por bando teve correlação positiva, com $r=0,8$. O tamanho médio dos bandos foi de 17,26 indivíduos e composição média de 6,71 espécies por bando, com alterações significativas durante o ano, sendo os bandos maiores e mais ricos em espécies na estação mais úmida (novembro a março).

Palavras-chave: bandos mistos de aves, Mata Atlântica, comportamento de forrageamento.

\section{INTRODUÇÃO}

Diferentes indivíduos de uma comunidade de aves podem associar-se por diversos fatores. Bando misto é um tipo de associação que se man- tém e é causada por respostas comportamentais mútuas entre seus integrantes (Moynihan, 1962).

Indivíduos que participam de bandos podem ser beneficiados pela maximização de forrageio (Morse, 1970; Murton, 1971), diminuição dos 
riscos de predação (Hamilton, 1971; Lazarus, 1972; Willis, 1972) ou ambas (Moynihan, 1962; Powell, 1985).

A sazonalidade climática e reprodutiva influencia o número de indivíduos e de espécies nos bandos, a freqüência de ocorrência dos bandos (Moynihan, 1962; Munn, 1985; Powell, 1985; Morrison et al., 1987; Machado, 1991) e sua composição, através da diminuição da participação de diversas espécies durante seu período reprodutivo e do ingresso de espécies migrantes (Moynihan, 1962; Powell, 1985; Alves, 1988; Machado, 1997).

No Brasil são escassos os estudos sobre bandos mistos, havendo registros no Cerrado do Planalto Central (Silva, 1980; Alves, 1988) e na Mata Atlântica na região de Teresópolis (RJ), por Davis (1946), e na Serra de Paranapiacaba, por Machado (1991, 1997).

Este estudo amplia os dados disponíveis sobre este tipo de associação na Mata Atlântica do alto da Serra de Paranapiacaba, através da análise da dinâmica dos bandos e da sua composição específica.

\section{MATERIAL E MÉTODOS}

Este estudo foi conduzido no Parque Estadual Intervales - PEI $\left(24^{\circ} 12^{\prime}\right.$ a $24^{\circ} 25^{\prime} \mathrm{S} ; 4^{\circ} 03^{\prime}$ ' a $48^{\circ} 30^{\prime} \mathrm{W}$ ), Ribeirão Grande, SP, Brasil. O PEI localiza-se na Serra de Paranapiacaba, possuindo uma área de 49000 ha, fazendo fronteiras com outras áreas de conservação, totalizando assim 120000 ha contínuos de área de Mata Atlântica preservada.

No PEI predomina o relevo do tipo "montanha com vales profundos", sustentado por rochas graníticas e calcáreas (Ponçano, 1981). Os dados foram obtidos em altitude média de $950 \mathrm{~m}$. $\mathrm{O}$ clima na área de estudo é classificado como $\mathrm{Cfb}$, segundo Köepen; as chuvas ocorrem durante o ano todo e são de caráter orográfico, podendo-se determinar uma estação úmida (de outubro a março) e outra subseca (de abril a setembro) (Rodrigues et al., 1994a). A precipitação anual é de cerca de $1800 \mathrm{~mm}$, podendo ocorrer geadas nos meses de junho a agosto. A temperatura média anual é de cerca de $22^{\circ} \mathrm{C}\left(15^{\circ} \mathrm{C}\right.$ na estação subseca e $25^{\circ} \mathrm{C}$ na estação úmida), com mínima registrada de $2{ }^{\circ} \mathrm{C}$ e máxima de $32{ }^{\circ} \mathrm{C}$ (Machado, 1997).

A Mata Atlântica é definida por AndradeLima (1966) como "floresta perenifólia latifoliada higrófila costeira". Caracteriza-se por grandes concentrações arbóreas distribuídas em dois ou mais estratos, onde desenvolvem-se numerosas epífitas e lianas herbáceas e lenhosas. Observa-se uma heterogeneidade na vegetação no PEI, devido a áreas em diferentes fases de desenvolvimento sucessional, ocorrendo áreas de vegetações primárias, secundárias antigas e mais recentes (para outros detalhes sobre a vegetação local, veja Nascimento, 1994).

Os dados foram obtidos no período de abril de 1990 a março de 1991, realizando-se mensalmente uma expedição de seis dias, com uma amostragem de seis horas em cada dia, sendo que $60 \%$ destas foram iniciadas logo ao nascer do sol, totalizando 432 horas de campo.

Em cada expedição, caminhava-se cerca de $40 \mathrm{~km}$ nas trilhas e estradas do parque. A irregularidade do terreno e a presença de um sub-bosque geralmente muito denso impossibilitavam que bandos mistos de aves, quando contatados, fossem seguidos por muito tempo, a não ser quando estes se deslocavam junto às trilhas. As observações foram feitas com auxílio de binóculos 7 X 35 .

Usou-se a definição proposta por Herrera (1979) sendo considerado bando misto qualquer associação de dois ou mais indivíduos de espécies diferentes que se deslocam juntos e com sincronicidade. Ao contatar-se um bando misto, registrava-se em gravador de bolso os seguintes dados: hora e tempo de contato (período de tempo entre o primeiro ao último indivíduo observado); composição específica; número aproximado de indivíduos de cada espécie; distribuição específica nos estratos da vegetação; posicionamento das espécies dentro do bando, definindo-se como área nuclear a região de maior densidade de indivíduos no bando, e como área periférica a região onde a densidade de indivíduos é menor; e tamanho aproximado do bando (em número de indivíduos).

A partir do número de contatos feitos com cada espécie, foi determinada a freqüência com que estas espécies ocorrem em bandos mistos, sendo classificadas em quatro categorias de freqüência (Powell, 1985): espécies regulares, comuns, pouco comuns e raras. Espécies regulares são as que estão presentes em mais de $25 \%$ dos bandos; espécies comuns têm freqüência de participação nos bandos de 10,0 a $24,99 \%$; as pouco comuns são as presentes de 3,0 a 9,99\% dos bandos; e são consideradas raras as que foram vis- 
tas em até $2,99 \%$ dos bandos. As espécies de aves seguiram a classificação taxonômica apresentada em Sick (1997).

Nem sempre todos os contatos tiveram registros completos do bando, devido a variáveis como altura, largura, velocidade e tamanho do bando, além de limitações visuais decorrentes da densidade do sub-bosque e nebulosidade (neblina ou chuva).

\section{RESULTADOS}

A variação diária de ocorrência de bandos mistos foi calculada pelo número de contatos com bandos para cada período de duas horas, sendo estes períodos consecutivos, contados a partir do nascer do sol (hora zero). Durante o dia, observou-se dois picos de maior freqüência de contatos com os bandos: o primeiro nas duas primeiras horas da manhã (freqüência de 1,0 bando/hora), e o segundo durante a tarde, entre oito a dez horas após o nascimento do sol ( 0,84 bando/hora). O período em que se teve menos contatos com bandos mistos foi entre seis e oito horas a partir do nascer do sol ( 0,36 bando/hora).

A variação de ocorrência mensal de bandos mistos foi calculada pela razão entre o número de bandos contatados a cada mês e o total mensal de horas de campo. Bandos mistos ocorreram durante o ano todo no PEI (Fig. 1). Nos meses de maio a outubro os bandos foram muito freqüentes, com pico no mês de agosto, no qual a razão chegou a 1,27 bando por hora de campo. Neste período, os bandos eram muito coesos, com os indivíduos próximos uns dos outros. A partir de novembro, ocorreu um decréscimo na freqüência de bandos, chegando a uma razão de 0,26 bando por hora em janeiro. Durante os meses de verão, mais chuvosos, os bandos eram pouco coesos e, em geral, ocupavam uma área de cobertura maior, com os indivíduos mais distantes entre si; não foi raro observar bandos se desagregarem, ou por divisão (dois ou mais grupos de aves seguiam direções diferentes) ou por diluição (o bando se fragmentava).

No PEI foram observados bandos formados por um mínimo de duas e no máximo 28 espécies de aves. A média de espécies por bando foi 6,71 $( \pm 4,74 ; N=388)$, mantendo-se estável de abril a novembro ( $x=6,04$ espécies por bando). Nos meses de verão houve um aumento no número de espécies por bando $(x=10,02)$, sendo esta diferença significativa $(t=6,02 ; \mathrm{P}<0,01)$ (Fig. 1).

O número de espécies por bando teve alta correlação positiva com o tamanho dos bandos ( $r=$ $0,80 ; N=388)$. A média de tamanho de bandos foi 17,26 indivíduos $( \pm 14,38 ; \mathrm{N}=388)$, com extremos de 2 a 120 indivíduos. A variação do tamanho médio de bandos é mostrada na Fig. 2.

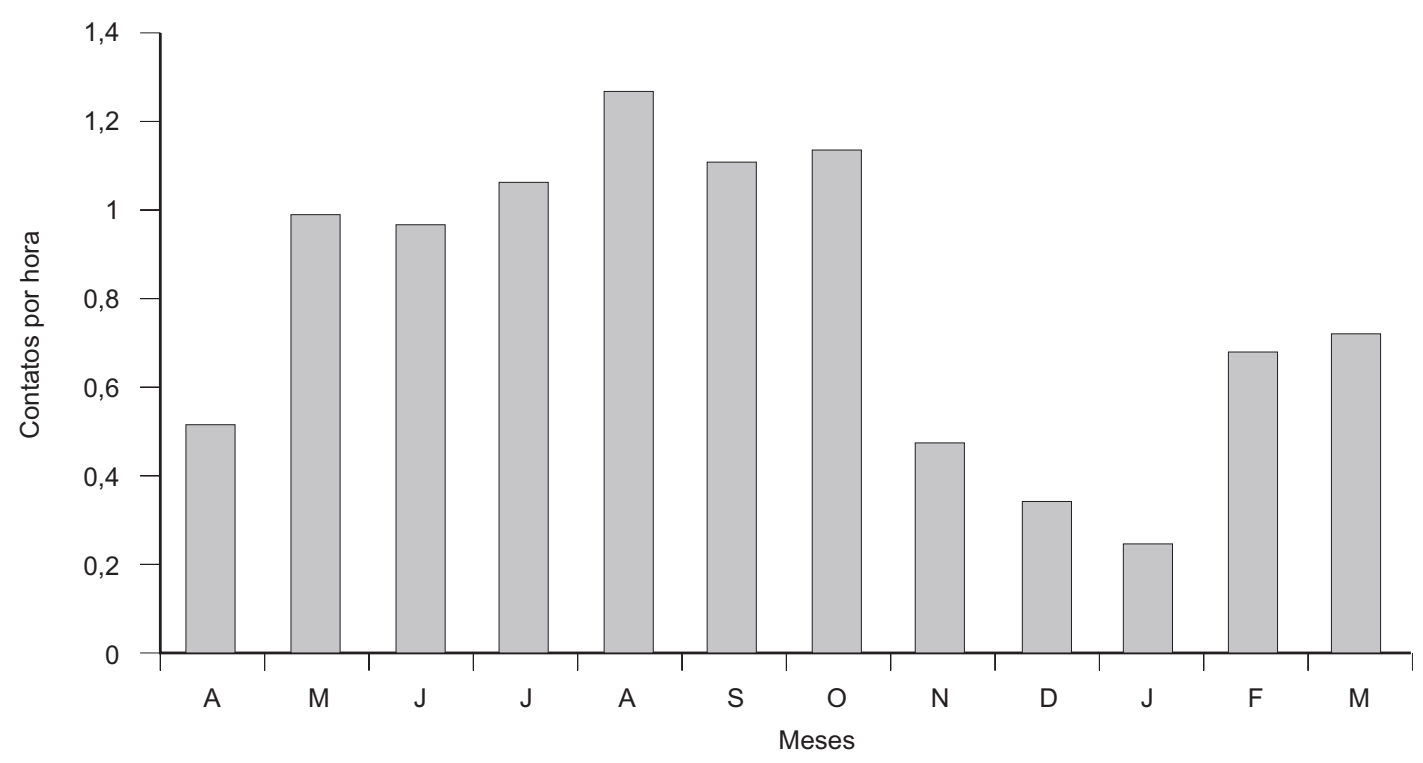

Fig. 1 - Variação mensal de ocorrência de contatos com bandos mistos por hora de campo na Mata Atlântica do Parque Estadual Intervales, no sudeste brasileiro. 


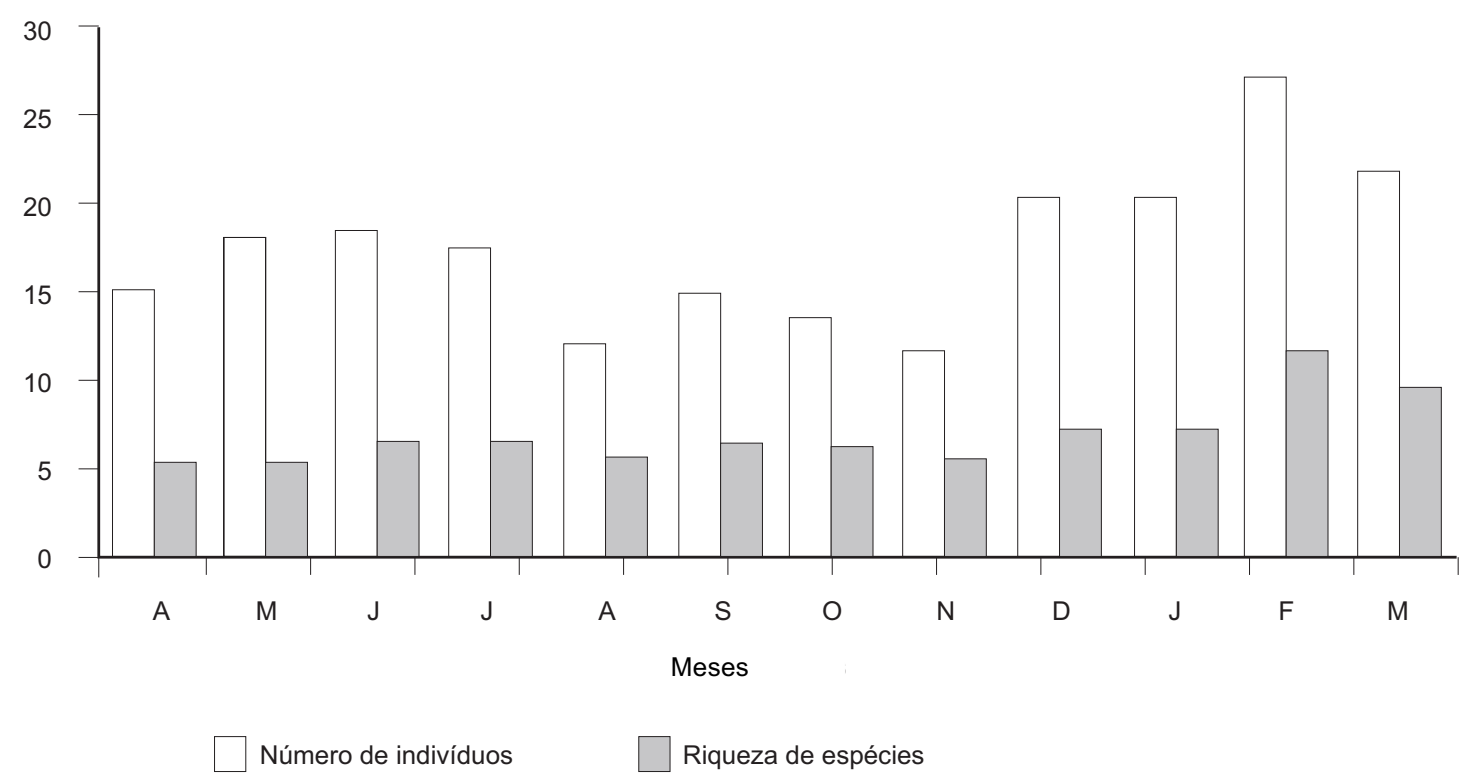

Fig. 2 - Variação da riqueza de espécies e do tamanho de bandos mistos (em número de indivíduos) na Mata Atlântica do Parque Estadual Intervales, no sudeste brasileiro.

Foram feitos 388 contatos com bandos mistos, registrando-se um total de 120 espécies de aves integrando estes grupos (Tabela 1).

Apenas Basileuterus culicivorus, Philydor rufus e Sittasomus griseicapillus foram categorizadas como espécies regulares nos bandos. Dezessete espécies foram classificadas como comuns. À exceção de Vireo olivaceus (espécie migratória), todas são residentes locais. A categoria de espécies pouco comuns contém 36 espécies, sendo que algumas são residentes e outras apresentam deslocamentos populacionais. O grupo de espécies raras corresponde a 53,3\% do total de espécies registradas integrando bandos mistos.

A Tabela 1 mostra o posicionamento de cada espécie nos estratos da vegetação, quando em bandos mistos.

Dentre as treze espécies de aves não passeriformes observadas em bandos mistos no PEI, apenas três (psitacídeos e buconídeos) tem participação rara e efêmera nos bandos, sendo sua associação provavelmente acidental. Os tucanos e araçaris (Ramphastidae), embora raros em bandos mistos, seguem-nos em suas áreas periféricas. Piaya cayana (Cuculidae), Trogon surrucura e $T$. viridis (Trogonidae) apresentam-se coesos com os bandos mistos, seguindo-os sós ou aos pares na periferia dos bandos, movimentando-se e vocalizando pouco. P. cayana forrageia capturando insetos no ar, em vôo ou sobre ramos, e os trogonídeos se alimentam de frutos, flores e insetos pegos no ar, em curtos vôos.

Os pica-paus (Picidae) seguem freqüentemente os bandos mistos. São vistos aos pares, posicionando-se tanto em áreas periféricas como nucleares. Vocalizam pouco, com exceção de Melanerpes flavifrons, que pode ser ouvido à distância.

As quatorze espécies de tamnofilídeos são vistas aos pares (exceto Terenura maculata e Pyriglena leucoptera, que ocorrem em pequenos grupos homoespecíficos), distribuindo-se de maneira heterogênea na vegetação (Tabela 1). Ocupam, de forma geral, a área nuclear dos bandos.

Os furnarídeos, geralmente, são encontrados aos pares. Movimentam-se e vocalizam intensamente. Forrageiam em troncos (cascas ou musgos), ramos e folhas verdes ou secas.

Distribuem-se tanto na área nuclear como na periferia dos bandos. Phylidor rufus é a segunda espécie mais freqüente em bandos mistos, e é vista em agrupamentos homoespecíficos de até 20 indivíduos ( $x=4,32 \pm 3,46$ indivíduos). Movimenta-se intensamente, examinando musgos nos troncos, folhas secas ou verdes, pontas de ramos, cipós, inflorescências e rosetas de bromélias no subbosque e também em copas. Ocorre nos bandos durante todo o ano. 
TABELA 1

Espécies de aves nos bandos mistos no Parque Estadual Intervales, posicionamento nos estratos da vegetação, freqüência de participação e categorização (segundo Powell, 1985) em 388 bandos registrados entre abril de 1990 e março de 1991.

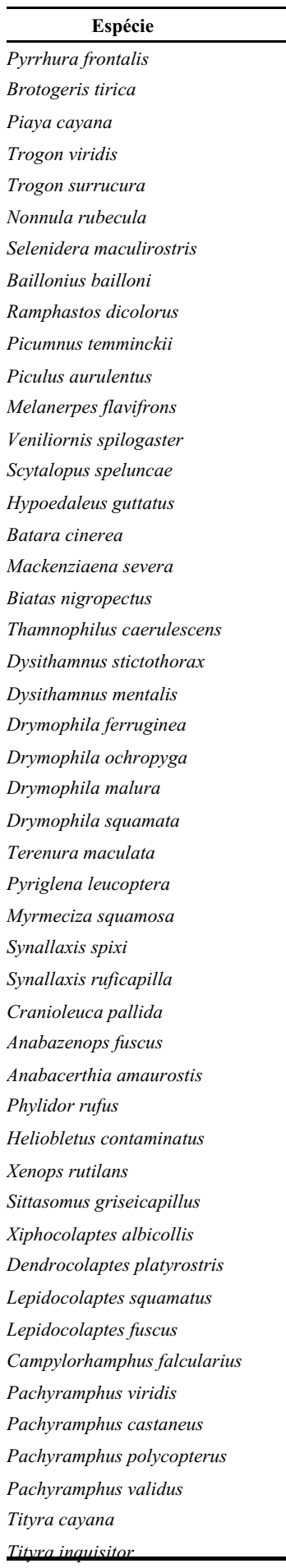

\begin{tabular}{|c|c|c|c|c|}
\hline Fam. & PV & $\%$ & Cat. & Espécie \\
\hline Psi & $5-6$ & 0,2 & RR & Phillomyias fasciatus \\
\hline Psi & $5-6$ & 1,0 & RR & Elaenia flavogaster \\
\hline Cuc & $4-5$ & 3,0 & $\mathrm{PC}$ & Serpophaga subcristata \\
\hline Tro & 4 & 2,8 & RR & Mionectes rufiventris \\
\hline Tro & 4 & 5,9 & $\mathrm{PC}$ & Leptopogon amaurocephalus \\
\hline Buc & $3-4$ & 0,2 & RR & Phylloscartes oustaleti \\
\hline Ram & $4-5$ & 0,2 & RR & Phylloscartes sylviolus \\
\hline Ram & $4-5$ & 1,2 & RR & Myiornis auricularis \\
\hline Ram & $4-5$ & 0,2 & RR & Hemitriccus obsoletus \\
\hline Pic & $1-5$ & 13,1 & $\mathrm{CO}$ & Todirostrum plumbeiceps \\
\hline Pic & $3-5$ & 4,1 & $\mathrm{PC}$ & Tolmomyas sulphurescens \\
\hline Pic & $4-6$ & 3,35 & $\mathrm{PC}$ & Platyrinchus mystaceus \\
\hline Pic & $4-5$ & 9,0 & $\mathrm{PC}$ & Myiobius barbartus \\
\hline Rhi & 1 & 0,5 & RR & Myiophobus fasciatus \\
\hline Tha & 2 & 0,2 & RR & Colonia colonus \\
\hline Tha & $1-2$ & 1,0 & RR & Muscipipra vetula \\
\hline Tha & $1-2$ & 1,2 & $\mathrm{RR}$ & Attila rufus \\
\hline Tha & 3 & 0,2 & RR & Serystes sibilator \\
\hline Tha & $1-3$ & 14,0 & $\mathrm{CO}$ & Myiarchus swainsoni \\
\hline Tha & 4 & 4,12 & $\mathrm{PC}$ & Pitangus sulphuratus \\
\hline Tha & 4 & 0,7 & RR & Megarynchus pitangua \\
\hline Tha & $1-2$ & 5,1 & $\mathrm{PC}$ & Myiozetetes similis \\
\hline Tha & $1-2$ & 2,5 & RR & Myiodynastes maculatus \\
\hline Tha & $1-2$ & 2,0 & RR & Tyrannus melancholicus \\
\hline Tha & $1-2$ & 1,5 & $\mathrm{RR}$ & Chiroxiphia caudata \\
\hline Tha & 5 & 0,7 & RR & Ilicura militaris \\
\hline Tha & $1-3$ & 2,0 & RR & Phibalura flavirostris \\
\hline Tha & $1-2$ & 0,5 & RR & Capornis cucullatus \\
\hline Fur & 1 & 0,7 & RR & Oxyruncus cristatus \\
\hline Fur & 1 & 16,7 & $\mathrm{CO}$ & Lipaugus lanioides \\
\hline Fur & $3-4$ & 5,1 & $\mathrm{PC}$ & Piprites chloris \\
\hline Fur & $2-4$ & 8,7 & $\mathrm{PC}$ & Troglodytes aedon \\
\hline Fur & 3 & 3,0 & $\mathrm{PC}$ & Platicichla flavipes \\
\hline Fur & $1-6$ & 34,5 & $\mathrm{RG}$ & Turdus rufiventris \\
\hline Fur & $2-4$ & 5,4 & $\mathrm{PC}$ & Turdus amaurochalinus \\
\hline Fur & $3-4$ & 9,7 & $\mathrm{PC}$ & Cyclarhis gujanensis \\
\hline Den & $2-5$ & 31,7 & $\mathrm{RG}$ & Vireo olivaceus \\
\hline Den & $2-5$ & 2,0 & $\mathrm{RR}$ & Hylophilus poicilotis \\
\hline Den & $2-6$ & 5,6 & $\mathrm{PC}$ & Parula pitiayumi \\
\hline Den & $2-5$ & 9,2 & $\mathrm{PC}$ & Geothlypis aequinoctialis \\
\hline Den & $2-5$ & 3,8 & $\mathrm{PC}$ & Basileuterus culicivorus \\
\hline Den & $2-4$ & 0,2 & RR & Coereba flaveola \\
\hline Tyr & $4-5$ & 1,8 & RR & Orchesticus abeillei \\
\hline Tyr & $3-5$ & 21,1 & $\mathrm{CO}$ & Cissopis leveriana \\
\hline Tyr & $4-5$ & 2,5 & RR & Pyrrhocoma ruficeps \\
\hline Tyr & $4-5$ & 5,1 & $\mathrm{PC}$ & Hemithraupis ruficapilla \\
\hline Tyr & $5-6$ & 2,3 & RR & Orthogonys chloricterus \\
\hline Tyr & $5-6$ & 3.0 & $\mathrm{PC}$ & Tachyphonus coronatus \\
\hline
\end{tabular}

\begin{tabular}{|c|c|c|c|}
\hline Fam. & PV & $\%$ & Cat. \\
\hline Tyr & $2-3$ & 0,5 & $\mathrm{RR}$ \\
\hline Tyr & $3-5$ & 3,0 & $\mathrm{PC}$ \\
\hline Tyr & $4-5$ & 2,5 & $\mathrm{RR}$ \\
\hline Tyr & $2-3$ & 10,5 & $\mathrm{CO}$ \\
\hline Tyr & $4-5$ & 8,7 & $\mathrm{PC}$ \\
\hline Tyr & $4-5$ & 13,6 & $\mathrm{CO}$ \\
\hline Tyr & $4-5$ & 3,3 & $\mathrm{PC}$ \\
\hline Tyr & $2-4$ & 21,1 & $\mathrm{CO}$ \\
\hline Tyr & $2-3$ & 0,2 & $\mathrm{RR}$ \\
\hline Tyr & 2 & 12,8 & $\mathrm{CO}$ \\
\hline Tyr & $2-3$ & 2,3 & $\mathrm{RR}$ \\
\hline Tyr & $2-3$ & 3,8 & $\mathrm{PC}$ \\
\hline Tyr & $2-3$ & 1,0 & $\mathrm{RR}$ \\
\hline Tyr & $2-3$ & 0,2 & RR \\
\hline Tyr & 6 & 0,5 & RR \\
\hline Tyr & 6 & 2,3 & $\mathrm{RR}$ \\
\hline Tyr & $4-5$ & 0,5 & $\mathrm{RR}$ \\
\hline Tyr & $4-5$ & 3,0 & $\mathrm{PC}$ \\
\hline Tyr & $4-5$ & 7,7 & $\mathrm{PC}$ \\
\hline Tyr & $5-6$ & 1,5 & $\mathrm{RR}$ \\
\hline Tyr & $5-6$ & 3,3 & $\mathrm{PC}$ \\
\hline Tyr & 5 & 1,0 & $\mathrm{RR}$ \\
\hline Tyr & $4-5$ & 5,1 & $\mathrm{PC}$ \\
\hline Tyr & $4-5$ & 0,5 & $\mathrm{RR}$ \\
\hline Pip & $2-3$ & 3,0 & $\mathrm{PC}$ \\
\hline Pip & $2-3$ & 1,0 & $\mathrm{RR}$ \\
\hline Cot & $4-5$ & 0,5 & $\mathrm{RR}$ \\
\hline Cot & $4-5$ & 1,0 & $\mathrm{RR}$ \\
\hline Cot & 6 & 2,5 & $\mathrm{RR}$ \\
\hline Cot & $4-5$ & 1,0 & $\mathrm{RR}$ \\
\hline Cot & 3 & 2,3 & $\mathrm{RR}$ \\
\hline Trd & $1-3$ & 1,0 & $\mathrm{RR}$ \\
\hline Mus & $5-6$ & 0,7 & $\mathrm{RR}$ \\
\hline Mus & $2-3$ & 5,9 & $\mathrm{PC}$ \\
\hline Mus & $3-4$ & 0,2 & $\mathrm{RR}$ \\
\hline Vir & $3-4$ & 13,4 & $\mathrm{CO}$ \\
\hline Vir & $3-5$ & 16,7 & $\mathrm{CO}$ \\
\hline Vir & $3-5$ & 17,0 & $\mathrm{CO}$ \\
\hline $\mathrm{Emb}$ & $3-4$ & 10,8 & $\mathrm{CO}$ \\
\hline $\mathrm{Emb}$ & $1-2$ & 2,3 & RR \\
\hline Emb & $1-5$ & 43,8 & $\mathrm{RG}$ \\
\hline Emb & 3 & 0,2 & $\mathrm{RR}$ \\
\hline Emb & 4 & 0,2 & RR \\
\hline Emb & $3-6$ & 4,8 & $\mathrm{PC}$ \\
\hline Emb & 5 & 0,2 & $\mathrm{RR}$ \\
\hline Emb & $4-6$ & 6,9 & $\mathrm{PC}$ \\
\hline Emb & $2-6$ & 8,5 & $\mathrm{PC}$ \\
\hline Emb & $1-3$ & 12.1 & $\mathrm{CO}$ \\
\hline
\end{tabular}


TABELA 1 (continuação)

\begin{tabular}{|c|c|c|c|c|c|c|c|c|c|}
\hline Espécie & Fam. & PV & $\%$ & Cat. & Espécie & Fam. & PV & $\%$ & Cat. \\
\hline Habia rubica & $\mathrm{Emb}$ & $1-2$ & 0,5 & RR & Dacnis cayana & $\mathrm{Emb}$ & $2-5$ & 2,5 & $\mathrm{RR}$ \\
\hline Thraupis cyanoptera & $\mathrm{Emb}$ & $4-6$ & 7,4 & $\mathrm{PC}$ & Haplospiza unicolor & $\mathrm{Emb}$ & $2-4$ & 1,5 & $\mathrm{RR}$ \\
\hline Thraupis ornata & $\mathrm{Emb}$ & $4-5$ & 4,3 & $\mathrm{PC}$ & Donascopiza albifrons & $\mathrm{Emb}$ & $1-2$ & 0,2 & $\mathrm{RR}$ \\
\hline Pipraeidea melanonota & $\mathrm{Emb}$ & $3-4$ & 3,3 & $\mathrm{PC}$ & Sicalis flaveola & $\mathrm{Emb}$ & 1 & 0,2 & $\mathrm{RR}$ \\
\hline Euphonia violacea & $\mathrm{Emb}$ & $3-4$ & 0,7 & $\mathrm{RR}$ & Volatinia jacarina & Emb & 1 & 0,5 & $\mathrm{RR}$ \\
\hline Euphonia pectoralis & $\mathrm{Emb}$ & $3-5$ & 4,8 & $\mathrm{PC}$ & Pitylus fulginosus & Emb & 3 & 1,5 & $\mathrm{RR}$ \\
\hline Chlorophonia cyanea & $\mathrm{Emb}$ & $3-5$ & 0,7 & RR & Saltator similis & $\mathrm{Emb}$ & $2-5$ & 12,3 & $\mathrm{CO}$ \\
\hline
\end{tabular}

Fam.: Família - PSI: Psittacidae; Cuc: Cuculidae; Tro: Trogonidae; Buc: Bucconidae; Ram: Ramphastidae; Pic: Picidae; Rнг: Rhinocryptidae; Тна: Thamnophilidae; Fur: Furnariidae; Den: Dendrocolaptidae; Tyr: Tyrannidae; Pip: Pipridae; Сот: Cotingidae; TRD: Troglodytidae; Mus: Muscicapidae; VIR: Vireonidae; Emb: Emberizidae.

PV: Posicionamento nos estratos da vegetação. 1 - porção inferior do sub-bosque; 2 - porção média do sub-bosque; 3 - porção superior do sub-bosque; 4 - porção inferior da copa; 5 - porção média da copa e 6 - porção superior da copa.

\% : Freqüência relativa de contatos nos bandos $(\mathrm{N}=388)$.

Cat:: Categoria de freqüência - RG: espécie regular (mais de 25,0\%); CO: espécie comum (de 10 a 24,99\%); PC: espécie pouco comum (de 3 a 9,99\%); e RR: espécie rara (até 2,99\%).

Os arapaçus (Dendrocolaptidae) são vistos aos pares, tanto na periferia como na área nuclear dos bandos, forrageando sob cascas de troncos e galhos; Lepidocolaptes ssp. e Dendrocolaptes platyrostris também examinam rosetas de bromélias epífitas. Sittasomus griseicapillus, espécie regular, ocorre nos bandos durante o ano todo.

Os tiranídeos são muito comuns nos bandos. Ocorrem aos pares ou em grupos homoespecíficos (Phylloscartes oustaleti). Predominantemente insetívoros, capturam suas presas em vôos curtos. Distribuem-se de maneira heterogênea nos estratos da vegetação (Tabela 1 ).

Os piprídeos e os cotingídeos ocupam tanto áreas nucleares como periféricas dos bandos, alimentando-se de pequenos frutos e insetos, pegos em vôos rápidos e curtos. Já os sabiás (Muscicapidae) situam-se em áreas periféricas dos bandos, sós ou aos pares, capturando artrópodos em troncos, galhos e folhas, alimentando-se também de frutos. Os vireonídeos são muito freqüentes em bandos mistos. Ocorrem aos pares, sendo que Hylophilus poicilotis e Vireo olivaceus ocupam área nuclear e Cyclarhis gujanensis posiciona-se na periferia dos bandos.

A Família Emberizidae é a que mais contribui em número de espécies nos bandos mistos: 34 espécies. Basileuterus culicivorus (subfamília Parulinae) é a espécie mais freqüente.

Geralmente aos pares, B. culicivorus movimenta-se muito, examinando as superfícies superior e inferior de folhas verdes, ramos, cipós, eventualmente, capturando insetos sobre folhas e no ar, em vôo. Vocaliza intensamente, emitindo pequenos gritos roucos e um canto curto e agudo. É freqüente durante todo o ano (Fig. 3) posicionando-se tanto na área nuclear como na periférica dos bandos.

Todas as espécies das subfamílias Thraupinae e Cardinalinae mostram grande habilidade em participar de associações heteroespecíficas. São encontradas aos pares ou em pequenos grupos homoespecíficos, como Orthogonys chloricterus ( $x=5,92 \pm 3,92$ indivíduos), Cissopis leveriana $(x=3,13 \pm 1,12)$, Tangara desmaresti $(x=10,88 \pm$ $5,41), T$. cyanocephala $(x=7,33 \pm 5,71)$ e $T$. seledon $(x=5,42 \pm 5,77)$. Com exceção de Donacospiza albifrons e Haplospiza unicolor, as espécies da subfamília Emberizinae integram bandos apenas em áreas próximas a campos de cultivo. Situam-se nas áreas periféricas dos bandos, sendo que apenas Zonotrichia capensis e Poospiza lateralis foram observadas aos pares e as demais, em grupos homoespecíficos de 5 a 15 indivíduos. 


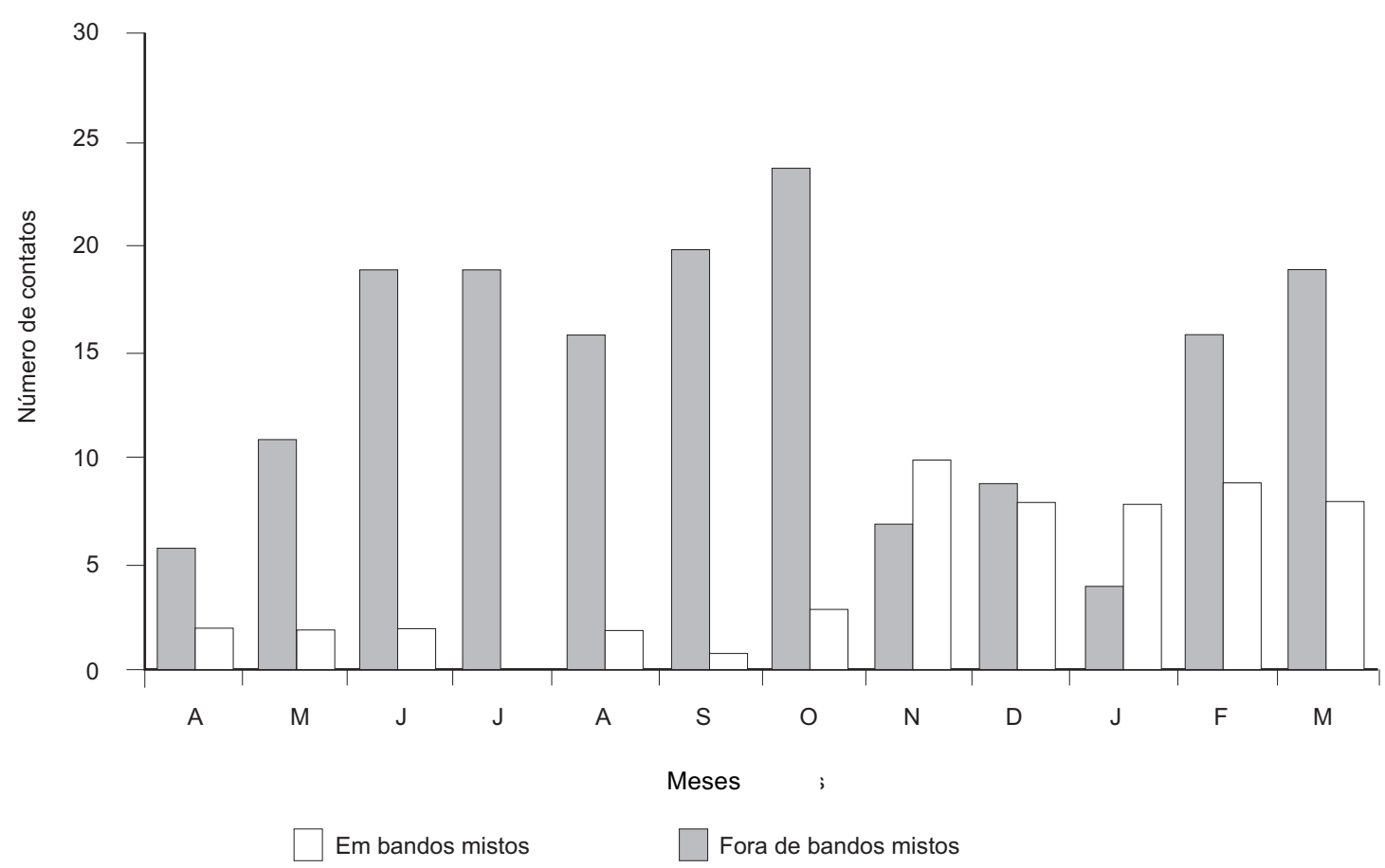

Fig. 3 - Número de contatos mensais, dentro e fora de bandos mistos, com Basileuterus culicivorus, espécie regular nos bandos mistos (presente em mais de $25 \%$ dos bandos) da Mata Atlântica do Parque Estadual Intervales, no sudeste brasileiro.

Dentre os emberezídeos, os icteríneos vocalizam constantemente, sendo ouvidos a longas distâncias. Capturam artrópodos em ramos, folhas e em ninhos de lagartas; comem sementes de Cassia spp. e Cedrela fissilis, abrindo os frutos secos com o bico. Cacicus chrysopterus é comumente visto em bandos mistos e aos pares e C. haemorrhous é sempre visto em grupos homoespecíficos de até cinco indivíduos.

\section{DISCUSSÃO}

A variação diária de ocorrência de bandos é semelhante à observada por Buskirk et al. (1972), Munn \& Terborgh (1979), Powell (1979), Alves (1988) e Laman (1992). Os bandos mistos podem ser vistos durante todo o dia, sendo mais freqüentes pela manhã e ao fim da tarde, períodos em que, geralmente, as aves estão mais ativas em busca de alimentos (Sick, 1997).

A variação da freqüência de ocorrência de bandos mistos no decorrer do ano (Fig. 1) segue o padrão encontrado em outras áreas estudadas (Davis, 1946; Moynihan, 1962; Morse, 1970;
Powell, 1979, 1985; Munn \& Terborgh 1979; Silva, 1980; Munn, 1985; Alves, 1988; Laman 1992), sendo que esta variação pode ser atribuída a dois fatores: maximização do forrageio e período reprodutivo.

Durante a estação subseca, de abril a setembro, os bandos são bastante freqüentes. Embora no PEI inexistam estudos que descrevam flutuações populacionais de artrópodos, em matas semidecíduas, dentro do domínio da Mata Atlântica, Ferrari (1986) encontrou alta correlação positiva entre a abundância de insetos e pluviosidade mensal. As baixas temperaturas e geadas registradas no PEI, concentradas no inverno, são fenômenos que também provavelmente causam queda populacional em artrópodos.

Com a diminuição da disponibilidade de presas potenciais, o aumento da participação em bandos mistos é uma estratégia vantajosa para muitas espécies de aves, que garantem o suprimento necessário de alimento, capturando insetos crípticos que são espantados pelo distúrbio causado pelo bando (Munn \& Terborgh, 1979; Rodrigues et al., 1994b). 
Além disso, este é o período inter-reprodutivo da maioria das aves da região (Sick, 1997), sendo que seus territórios não são estritamente delimitados, podendo juntar-se aos bandos por mais tempo (Powell, 1985). Nos meses mais quentes, durante a estação úmida (de outubro a março), aumenta a disponibilidade de alimento e mesmo com a presença de espécies de aves migratórias (Machado, 1997) os recursos são abundantes, sendo mais fácil o forrageio individual.

Em seu período reprodutivo, as aves tendem a participar menos dos bandos mistos (Fig. 3) (Moynihan, 1962; Morse, 1970, Powell, 1985). Neste estudo, foram observadas diversas espécies que participam de bandos mistos em atividades reprodutivas, como corte, construção de ninho e alimentação de ninhegos.

No PEI, bandos mistos são compostos, principalmente, por pares de indivíduos de cada espécie. O número de indivíduos por bando (tamanho) versus o número de espécies (riqueza) por bando tem alta correlação positiva, como tem sido observado em ambientes tropicais (Alves, 1988; Powell, 1979). Corroborando Powell (1985), também no PEI o tamanho dos bandos não aumenta pelo ingresso de mais indivíduos de cada espécie, e sim pela adição de novas espécies no bandos.

O número médio de espécies por bando é próximo ao valor encontrado por Davis (1946) na Mata Atlântica, em Teresópolis. No entanto, durante o período reprodutivo, surpreendentemente, houve um significativo aumento do número médio de espécies por bando. Paralelamente, o tamanho dos bandos acompanhou este aumento no número de espécies.

Desta forma, na estação úmida os bandos apresentaram-se mais ricos em espécies e, conseqüentemente, maiores, o que não é comum, pois em áreas de elevações médias nos trópicos, a redução do tamanho dos bandos e do número de espécies neste período é mais pronunciada que em áreas de pequenas elevações (Powell, 1985). Porém, fato similar pôde ser observado em outra área da Mata Atlântica por Davis (1946), onde nos meses de janeiro a abril verifica-se um aumento tanto no número de espécies por bando quanto no tamanho dos bandos.

Não é válido afirmar que este aumento ocorra pela presença de espécies migratórias na região nos meses de primavera e verão. Fosse esta alteração causada pelo ingresso destas espécies na comunidade, deveria se observar um aumento significativo no número médio de espécies por bando a partir do mês de setembro, quando estas espécies chegam à área, o que de fato não ocorre. Além disso, espécies migratórias tendem a participar em menor freqüência nos bandos ao entrarem em período reprodutivo, como se verifica em Vireo olivaceus (Machado 1997).

Provavelmente, a variação ocorrida no número médio de espécies por bando e no tamanho destes, esteja simplesmente refletindo a variação mensal de ocorrência de bandos mistos (Fig. 1). Durante a estação reprodutiva, algumas espécies deixam de participar destes agrupamentos, porém outras apenas associam-se menos freqüentemente. Com uma baixa freqüência de ocorrência de bandos mistos neste período, pode haver uma maior concentração destas espécies nos poucos bandos formados, resultando em um maior número de espécies por bando e, conseqüentemente, um maior tamanho destes. Isto também explica/ os picos encontrados no número de espécies por bando e tamanho destes no estudo de Davis (1946), já que nestes meses a freqüência de contatos com bandos é baixa.

Entretanto, a relação inversamente proporcional entre freqüência de ocorrência e tamanho/ riqueza de bandos mistos na época reprodutiva não pode ser explicada unicamente pela variação numérica entre esses três componentes. Powell (1985) sugere que o decréscimo no tamanho de bandos e no número médio de espécies seja resultante do fato de que, em florestas tropicais de elevações médias, ocorre apenas uma espécie atuando como núcleo, cuja efetividade, como força coesiva de bandos, reduz-se durante seu período reprodutivo. $\mathrm{Na}$ ausência de uma espécie-núcleo alternativa, os bandos tenderiam a se desintegrar. Machado (1991) analisou detalhadamente cinco espécies mais relevantes nos bandos mistos do PEI e sugeriu que na área estudada não existe uma espécienúcleo típica e, sim um conjunto de espécies ("complexo-núcleo") que, juntamente, promove a formação e coesão de bandos mistos.

O número de espécies de aves que participam de bandos mistos varia em diferentes regiões dos trópicos (Powell, 1985). Neste estudo, foram observadas 120 espécies de aves nos bandos mistos no PEI (Tabela 1), o que corresponde à maior riqueza de espécies entre todos os estudos já realizados com este tipo de associação. 
Apesar da grande riqueza de espécies, o número de espécies regulares do PEI é inferior ao encontrado em outros estudos feitos em ambientes semelhantes. O número de espécies regulares varia de cinco a dez (Willis, 1966; Buskirk et al., 1972; Powell, 1979, 1985).

Das três espécies regulares observadas em bandos mistos no PEI, todas são também regulares nas áreas estudadas por Davis (1946): Basileuterus culicivorus (também a mais freqüente), Philydor rufus e Sittasomus griseicapillus.

A variação mensal de participação das espécies regulares em bandos mistos é semelhante. Nos meses mais frios, de maio a outubro, a ocorrência em bandos mistos é alta, com poucos ou nenhum contato feito com estas espécies fora destes bandos. Nos meses de novembro a abril, com médias de temperatura maiores, esta situação é alterada, havendo um decréscimo na freqüência de participação em bandos, sendo que algumas deixam de se associar nestes agrupamentos e, conseqüentemente, ocorre um aumento de contatos com estas espécies fora de bandos mistos (Fig. 3). De forma geral, este padrão pode ser observado entre a maioria das espécies comuns e pouco comuns. Para as espécies raras é difícil avaliar a sazonalidade de participação nos bandos, pois muitas vezes ocorrem em baixa densidade populacional, têm comportamento críptico (dificultando o contato) e/ou são pouco propensas ao gregarismo interespecífico (Powell, 1985).

As espécies de aves que não pertencem à ordem Passeriformes, registradas em bandos mistos na região do PEI, são poucas, geralmente raras, e não contribuem significativamente para a formação e coesão dos bandos. Dentro deste grupo, porém, algumas espécies são boas seguidoras de bandos mistos, como o cuculídeo Piaya cayana, os trogonídeos e os picídeos.

As duas espécies de Trogon obtêm clara vantagem na associação em bandos mistos: freqüentemente são vistos capturando, em vôo, insetos espantados pelo distúrbio causado por outros integrantes do bando. No PEI, apesar de raras nos bandos, essas duas espécies seguem estes bandos por longos períodos de tempo. Os picídeos são beneficiados, principalmente, na proteção contra predadores, pois alimentam-se de larvas presentes dentro de galerias, em troncos e galhos, e não de artrópodos espantados pelo bando. Com uma maior proteção contra predadores dentro dos bandos, conseqüentemente, pode haver uma otimização de forrageio, assim como em outras espécies, pois podem despender mais tempo à procura de alimento, já que, quando sozinhos, fora de bandos mistos, permanecem grande parte de tempo vigiando a presença de predadores (Willis, 1972; Sullivan, 1984).

Os Passeriformes representam $89 \%$ das espécies observadas nos bandos do PEI. Os tamnofilídeos têm sido registrados em bandos mistos em diversas áreas (Powell, 1985), sendo que, na região amazônica, algumas espécies (Thamnomanes spp.) são classificadas como espécies-núcleo (Munn \& Terborgh, 1979; Munn, 1985; Powell, 1985). Dysithamnus mentalis figura entre as espécies regulares nas áreas de Mata Atlântica estudadas por Davis (1946), sendo a segunda espécie mais freqüente em umas das áreas em que estudou. No entanto, no alto da Serra de Paranapiacaba é uma espécie rara nos bandos mistos, assim como a maioria dos tamnofilídeos.

Espécies da família Furnariidae são autênticas integrantes de bandos mistos, forrageando intensamente no sub-bosque (Rodrigues et al., 1994a), causando distúrbios locais, atraindo outras espécies. Synallaxis ruficapilla foi categorizada como espécie comum nos bandos do PEI, porém trata-se de uma espécie abundante na área de estudo, habitando o baixo sub-bosque, na borda de trilhas e estradas, podendo ter sido superestimada sua participação nos bandos.

Os dendrocolaptídeos são característicos de bandos mistos. Sittasomus griseicapillus e Lepidocolaptes fuscus são as espécies mais freqüentes desta família em bandos, também constatado por Davis (1946).

Os tiranídeos de forma geral, assim como os furnarídeos, contribuem significativamente para a formação e coesão dos bandos, pois grande parte de suas espécies vocalizam e movimentam-se muito, causando distúrbios no local e atraindo outras espécies. Algumas são verdadeiras integrantes de bandos mistos, como Pachyramphus castaneus, P. validus, Phylloscartes oustaleti, Platyrhychus mystaceus, Myiornis auricularis, Leptopogon amaurocephalus e Mionectes rufiventris. Esta última é considerada por Davis (1946) como espécie incidental, porém apresenta-se claramente integrada a estes agrupamentos, com padrão de movimentação, vocalização e deslocamento característico de participantes de bandos mistos. 
Os cotingídeos parecem associar-se por pouco tempo aos bandos, com raros contatos. No entanto, beneficiam-se claramente dos bandos, tendo sido observados diversas vezes alimentando-se de artrópodos espantados. Já os muscicapídeos são bastante integrados aos bandos. Turdus rufiventris apresenta alteração da altura média de forrageamento quando participa dos bandos (Machado, C.G. \& Rodrigues, N.M.R., in prep.), mostrando plasticidade em seu comportamento de forrageio para adequar-se aos bandos e deles obter vantagens. Os vireonídeos são membros típicos de bandos mistos. Permanecem associados aos bandos por longos períodos de tempo. Vireo olivaceus destaca-se por figurar entre as mais freqüentes em bandos mistos, apesar de estar presente na área apenas nos meses de primavera e verão (Machado, 1997).

De todas as famílias registradas, as espécies mais típicas estão entre os emberezídeos. O parulíneo Basileuterus culicivorus contribui muito na manutenção dos bandos, permanecendo integrado nestes por longos períodos de tempo. Dentro dos bandos, pares de $B$. culicivorus movimentam-se muito, deslocando-se em grande agito, examinando folhas e ramos. Machado (1991) sugere que $B$. culicivorus faça parte do conjunto de espécies responsáveis pela formação e coesão dos bandos no PEI. As espécies das subfamílias Thraupinae, Cardinalinae e Icterinae, embora de freqüências variadas, são verdadeiros membros de bandos mistos. Trichothraupis melanops é uma espécie claramente oportunista, aproveitando-se dos distúrbios causados por diferentes grupos de animais, como formigas de correição, macacos e bandos mistos de aves (Rodrigues et al., 1994b), para capturar os artrópodos em fuga. Habia rubica tem sido citada como uma espécie típica de bandos mistos de diversas localidades (Powell, 1985), sendo apontada como uma espécie-núcleo na Mata Atlântica na região da Juréia (Develey, 1996). No entanto, no PEI não é uma espécie abundante, tendo participação rara nos bandos mistos.

As espécies de Tangara são particularmente hábeis em participar de bandos mistos, muitas vezes em grupos congenéricos, altamente sincronizados em movimentos, velocidade e direção, parecendo constituir um grupo a parte do restante do bando onde estão inseridos. Mitchell (1957) sugere que Tangara cynocephala pode ser caracterizada como espécie-núcleo nestes bandos, porém os grupos compostos apenas por $T$. seledon e $T$. desmaresti também são coesos e sincronizados. As espécies da subfamília Emberizinae são todas raras, presentes nos bandos por curtos períodos de tempo.

Tanto Cacicus chrysopterus como C. haemorrhous são espécies tipicamente integradas aos bandos mistos. Pizo (1996) observou que, em bandos mistos, C. chrysopterus alimenta-se mais de artrópodos que quando fora de bandos, quando prevalecem itens vegetais em sua dieta, corroborando mais uma vez a idéia de que bandos mistos podem maximizar o forrageio por evidenciar artrópodos crípticos.

O Parque Estadual Intervales possui em torno de 320 espécies de aves (Silva, W.R., com. pess.). Aproximadamente, um terço destas aves foram observadas participando, em maior ou menor grau, em bandos mistos, o que denota a importância das associações heteroespecíficas na comunidade de aves. Desta forma, tais agrupamentos devem ser sempre detalhadamente estudados e considerados em projetos de conservação de espécies endêmicas e ameaçadas, assim como na conservação e manejo de áreas silvestres.

Agradecimentos - Agradeço à CAPES pelo o suporte financeiro; à Fundação Florestal, SP, pelo apoio logístico no Parque Estadual Intervales; aos companheiros dos Deptos. de Zoologia e Botânica da UNICAMP; aos Drs. Wesley R. Silva (pela orientação), Douglas F. Stotz, Edwin O. Willis, Jacques M.E. Vielliard, Emigdio L.A. Monteiro Filho, Marcos Rodrigues, Luiz O. Marcondes Machado e aos professores Marco A. Pizo e Nívea M.R. Rodrigues pelas valiosas críticas e sugestões a este trabalho.

\section{REFERÊNCIAS BIBLIOGRÁFICAS}

ALVES, M. A. S., 1988, Organização social e biologia reprodutiva de Neothraupis fasciata (Passeriformes: Thraupidae). Dissertação de Mestrado, Inst. Biol. da Univ. Est. Campinas, Campinas, SP, Brasil.

ANDRADE-LIMA, D., 1966, Vegetação. In: Atlas Nacional do Brasil, IBGE, Rio de Janeiro.

BUSKIRK, W. H., POWELL, G. V. N., WITTENBERGER, R. E. \& POWELL, T. U., 1972, Interspecific bird flocks in tropical highland Panama. Auk, 89: 612-624.

DAVIS, D. E., 1946, A seazonal analysis of mixed flocks of birds in Brazil. Ecology, 27: 168-181.

DEVELEY, P. F., 1996, Dados preliminares sobre o comportamento de Habia rubica (Emberizidae: Thraupinae) em uma área de Mata Atlântica, no Estado de São Paulo. Res. V Cong. Bras. Ornitol., 32. 
FERRARI, S., 1986, The behavior and ecology of the buffyheaded Marmoset, Callithrix flaviceps (O. Thomas, 1903). $\mathrm{PhD}$ Thesis, University of London, England.

HAMILTON, W. D., 1971, Geometry for the selfish herd. $J$. Theor. Biol., 31: 295-311.

HERRERA, C. M., 1979, Ecological aspects of heterospecifics flocks formation in a Mediterraniam passerine bird community. Oikos, 33: 85-96.

LAMAN, T. G., 1992, Composition o mixed-species foraging flocks in a Bornean Rainforest. Mal. Nat. Journ., 46(2): 131-144

LAZARUS, J., 1972, Natural selection and the functions of flocking in birds: a replay to Murton. Ibis, 114: 556-558.

MACHADO, C. G., 1991, Estrutura, composição e dinâmica de bandos mistos de aves na Mata Atlântica do alto da Serra de Paranapiacaba, SP. Dissertação de Mestrado, Inst. Biol. da Univ. Est. Campinas, Campinas, SP, Brasil.

MACHADO, C. G., 1997, Vireo olivaceus (Vireonidae, Aves): uma espécie migratória nos bandos mistos de aves da Mata Atlântica do sudeste brasileiro. Ararajuba, 5(1): 62-64.

MITCHELL, M. H., 1957, Observations on birds of southeastern Brazil. Toronto, University of Toronto Press.

MORRINSON, L., WITH, K. A., TIMOSSI, C. I. \& MILNE, K. A., 1987, Composition and temporal variation of flocks in the Sierra Nevada. Condor, 89: 739-745.

MORSE, D. H., 1970, Ecological aspects of some mixed-species foraging flocks of birds. Ecol. Monogr., 40: 119-168.

MOYNIHAN, M., 1962, The organization and probable evolution of some mixed-species flocks of neotropical birds. Smthson. Misc. Collect. 143: 1-140.

MUNN, C. A., 1985, Permanent canopy and understory flocks in Amazonia: species composition and population density. Ornith. Mononogr., 36: 683-712.

MUNN, C. A. \& TERBORGH, J. W., 1979, Multi-species territoriality in Neotropical foraging flocks. Condor, 81: 338-344.

MURTON, R. K., 1971, Why do some birds species feed in flocks? Ibis, 113: 534-536.
NASCIMENTO, F. H., 1994, A sucessão secundária inicial na Mata Atlântica sobre a Serra de Paranapiacaba, Ribeirão Grande, SP. Dissertação de Mestrado, Inst. Biociênc. Univ. São Paulo, São Paulo, Brasil.

PIZO, M. A., 1996, Feeding ecology of two Cacicus species (Emberizidae, Icterinae). Ararajuba, 4(2): 87-92.

PONÇANO, W. L., 1981, Mapa geomorfológico do Estado de São Paulo. Vol. 1, Secret. Ind. Com., Ciênc. e Tecn. Est. São Paulo, 94p.

POWELL, G. V. N., 1979, Structure and dynamics of interspecific flocks in a Neotropical mid-elevation forest. Auk, 96: 375-390.

POWELL, G. V. N., 1985, Sociobiology and adaptive significance of heterospecific foraging flocks in the Neotropics. Ornithol. Monogr., 36: 713-732.

RODRIGUES, M., ALVARES, S. M. R. \& MACHADO, C. G., 1994a, Forraging behavior of the white-collared foliage-gleaner (Anabazenops fuscus), a bamboo specialist. Ornit. Neotrop., 5: 65-67.

RODRIGUES, M., MACHADO, C. G., ALVARES, S. M. R. \& GALETTI, M., 1994b, Association of the BlackGoggled Tanager (Trichothraupis melanops) with Flushers. Biotrop., 26(4): 472-475.

SICK, H., 1997, Ornitologia Brasileira. Ed. Nova Fronteira, Rio de Janeiro, Brasil.

SILVA, E. M. D., 1980, Composição e comportamento de grupos heteroespecíficos de aves em área de cerrado, no Distrito Federal. Dissertação de Mestrado, Inst. Biol. da Univ. Brasília, Brasília, DF, Brasil.

SULLIVAN, K. A., 1984, Information exploitation by Downy Woodpeckers in mixed-species flocks. Behav., 91: 294311.

WILLIS, E. O., 1966, Competitive exclusion and birds fruiting trees in western Colombia. Auk, 83: 479-480.

WILLIS, E. O., 1972, Do birds flocks in Hawaii, a land without predators? Calif. Birds, 3(1): 1-8. 